\title{
ON THE ONE-DIMENSIONAL HARMONIC OSCILLATOR WITH A SINGULAR PERTURBATION
}

\section{V.A. Strauss, Departamento de Matemáticas Puras y Aplicadas, Universidad Simón} Bolívar, Caracas, Venezuela, str@usb.ve,

M.A. Winklmeier, Departamento de Matemáticas, Universidad de Los Andes, Bogotá, Colombia, mwinklme@uniandes.edu.co

In this paper we investigate the one-dimensional harmonic oscillator with a leftright boundary condition at zero. This object can be considered as the classical selfadjoint harmonic oscillator with a singular perturbation concentrated at one point. The perturbation involves the delta-function and/or its derivative. We describe all possible selfadjoint realizations of this scheme in terms of the above mentioned boundary conditions. We show that for certain conditions on the perturbation (or, equivalently, on the boundary conditions) exactly one non-positive eigenvalue can arise and we derive an analytic expression for the corresponding eigenfunction. These eigenvalues run through the whole negative semi-line as the perturbation becomes stronger. For certain cases an explicit relation between suitable boundary conditions, the non-positive eigenvalue and the corresponding eigenfunction is given.

Keywords: harmonic oscillator; singular perturbation; selfadjoint extensions; negative eigenvalues.

\section{Introduction}

In this paper, we use the following notations. Set $\mathbb{R}_{+}=(0, \infty)$ and $\mathbb{R}_{-}:=(-\infty, 0)$ and for functions $f: \mathbb{R} \rightarrow \mathbb{C}$ define their restrictions $f_{ \pm}:=\left.f\right|_{\mathbb{R}_{ \pm}}$. The standard inner products on $L_{2}(\mathbb{R})$ and on $\mathbb{C}^{n}$ are both denoted by $\langle\cdot, \cdot\rangle$. There will never be danger of confusion. Given a sesquilinear form $[\cdot, \cdot]$ on $\mathbb{C}^{n}$, we call a subspace $L$ neutral if $[u, v]=0$ for every $u, v \in L$. A subspace $L$ is called maximal neutral if it is neutral and not properly contained in any other neutral subspace (see [8] for details).

The one-dimensional harmonic oscillator is given by the formal differential expression

$$
\mathfrak{A} f(t):=\left(-\frac{1}{2} \frac{\mathrm{d}^{2}}{\mathrm{~d} t^{2}}+\frac{1}{2} t^{2}\right) f(t) .
$$

The aim of this paper is to investigate several possible realisations of $\mathfrak{A}$ as a symmetric linear operator and determine all possible selfadjoint extensions.

By the Liouville - Green asymptotic formula [5, Theorem 2.2.1], it is known that there are solutions $y_{ \pm}$of $\mathfrak{A} y=\lambda y$ with the following asymptotic behaviour for $|t| \rightarrow \infty$ :

$$
y_{ \pm}(t) \sim \frac{1}{\left(t^{2}-2 \lambda\right)^{\frac{1}{4}}} \exp \left( \pm \int_{a}^{t} \sqrt{s^{2}-2 \lambda+\frac{1}{4}\left(\frac{s}{s^{2}-2 \lambda}\right)^{2}} \mathrm{~d} s\right)
$$

for $|a|$ large enough. This shows immediately that $\mathfrak{A}$ is in the limit point case both at $+\infty$ and $-\infty$. 
In order to assign an operator to the differential expression $\mathfrak{A}$, we need to specify a domain of admissible functions. The minimal operator associated with $\mathfrak{A}$ is

$$
A^{\min } f=\mathfrak{A} f, \quad \mathcal{D}\left(A^{\min }\right)=C_{c}^{\infty}(\mathbb{R}) .
$$

Since $\mathfrak{A}$ is in the limit point case both at $+\infty$ and $-\infty$, the operator $A^{\text {min }}$ is essentially selfadjoint (see, e.g. [12, 7.1.3]). Its closure is the so-called maximal operator associated to $\mathfrak{A}:$

$$
A f=\mathfrak{A} f, \quad \mathcal{D}(A)=\left\{f: \mathbb{R} \rightarrow \mathbb{C}: f, f^{\prime} \text { abs. cont., } f, \mathfrak{A} f \in L_{2}(\mathbb{R})\right\} .
$$

Note that

$$
A^{\text {min }} \subset \overline{A^{\text {min }}}=A=A^{*} .
$$

It is well known that $A$ has a compact resolvent, and that its spectrum consists of simple eigenvalues:

$$
\sigma(A)=\sigma_{p}(A)=\left\{n+\frac{1}{2}: n \in \mathbb{N}_{0}\right\} .
$$

The corresponding eigenfunctions are

$$
\psi_{n}(t)=\mathrm{e}^{-t^{2} / 2} c_{n} H_{n}(t)
$$

where $H_{n}$ is the $n$th Hermite polynomial of order $n$,

$$
H_{n}(t)=(-1)^{n} \mathrm{e}^{t^{2}} \frac{\mathrm{d}^{n}}{\mathrm{~d} t^{n}} \mathrm{e}^{-t^{2}}
$$

and the normalization factor $c_{n}:=\left(\pi^{\frac{1}{2}} 2^{n} n !\right)^{-\frac{1}{2}}$ is chosen such that $\left\langle\psi_{n}, \psi_{m}\right\rangle=\delta_{n m}$.

Remark 1. A straightforward calculation shows that if $u$ is a solution of $(\mathfrak{A}+\lambda) u=0$, then $\left(t-\frac{\mathrm{d}}{\mathrm{d} t}\right)^{n} u$ is a solution of $(\mathfrak{A}+\lambda-n) u=0$ and $\left(t+\frac{\mathrm{d}}{\mathrm{d} t}\right)^{n} u$ is a solution of $(\mathfrak{A}+\lambda+n) u=0$.

In particular, all eigenfunctions of $A$ can be obtained by the recursion

$$
\psi_{0}(t)=\pi^{-\frac{1}{2}} \mathrm{e}^{-t^{2} / 2}, \quad \psi_{n}(t)=c_{n}\left(t-\frac{\mathrm{d}}{\mathrm{d} t}\right)^{n} \mathrm{e}^{-t^{2} / 2}, \quad n \geq 1 .
$$

Note that $\left(\frac{\mathrm{d}}{\mathrm{d} t}+t\right)^{n} \psi_{0}=0$, in agreement with the fact that $A$ has no negative eigenvalues.

From the recursion formula (5) it is clear that $\psi_{n}$ is an even function if $n$ is even, and that it is an odd function, if $n$ is odd.

In Section 1 we consider the restriction of the harmonic oscillator to the open half lines $\mathbb{R}_{ \pm}$:

$$
\begin{array}{ll}
A_{ \pm}^{\min } f(t):=\mathfrak{A} f(t), & \mathcal{D}\left(A_{ \pm}^{\min }\right):=C_{c}^{\infty}\left(\mathbb{R}_{ \pm}\right), \\
A_{ \pm}^{\max } f(t):=\mathfrak{A} f(t), & \mathcal{D}\left(A_{ \pm}^{\max }\right):= \begin{cases}\left.f: \mathbb{R}_{ \pm} \rightarrow \mathbb{C}: \begin{array}{l}
f, f^{\prime} \text { abs. cont. } \\
\left.\mathfrak{A} f\right|_{\mathbb{R}_{ \pm}} \in L_{2}\left(\mathbb{R}_{ \pm}\right)
\end{array}\right\}\end{cases}
\end{array}
$$

With them we define

$$
A_{0}=A_{-}^{\mathrm{min}} \oplus A_{+}^{\mathrm{min}} .
$$

Clearly, $\mathcal{D}\left(A_{0}^{*}\right)=\mathcal{D}\left(A_{-}^{\max }\right) \oplus \mathcal{D}\left(A_{+}^{\max }\right)$. 
In Sections 2 and 3 we will study several restrictions of the operator $A$ by imposing conditions on functions in its domain at $t=0$. We define the closed symmetric operators

$$
\begin{array}{ll}
B f:=\mathfrak{A} f, & \mathcal{D}(B):=\{f \in \mathcal{D}(A): f(0)=0\}, \\
C f:=\mathfrak{A} f, & \mathcal{D}(C):=\left\{f \in \mathcal{D}(A): f(0)=f^{\prime}(0)=0\right\}
\end{array}
$$

So we have the following chain of operators

$$
A_{0} \subset \overline{A_{0}}=C \subset B \subset A=A^{*} \subset B^{*} \subset C^{*}=A_{0}^{*} .
$$

With exception of the first one, all inclusions are one-dimensional. We will classify all selfadjoint extensions of $B$ (Section 2) and $C$ (Section 3) in terms of conditions on the behaviour at 0 of the functions in the corresponding domains. Slightly abusing language, we will call these conditions boundary conditions at 0 . We will not use the von Neumann extension theory for symmetric operators, but will identify selfadjoint extensions with maximal neutral subspaces of $\mathbb{C}^{2}, \mathbb{C}^{3}$ and $\mathbb{C}^{4}$, respectively, equipped with an inner product induced by the condition $\langle\mathfrak{A} f, g\rangle=\langle f, \mathfrak{A} g\rangle$ for $f, g$ in appropriate spaces.

In particular, it turns out that the selfadjoint extensions of $B$ can be parameterized by one real parameter. Every selfadjoint extension is of the form

$$
\mathcal{D}\left(B_{\theta}\right)=\left\{f \in \mathcal{D}\left(B^{*}\right): \sqrt{2} \cos (\theta) f(0)=\sin (\theta)\left[f^{\prime}(+0)-f^{\prime}(-0)\right]\right\}
$$

for $\theta \in[0, \pi)$. Any function of $\mathcal{D}\left(B_{\theta}\right)$ is continuous at 0 (Lemma 4 ), but its derivative has a jump proportional to the value of the function at 0 . There is a one-to-one correspondence between the constant of proportionality and the particular selfadjoint extension. The operators $B_{\theta}$ can also be interpreted as the classical harmonic oscillator with a $\delta$-interaction at 0 on a bigger Hilbert space, see Section 4:

$$
B_{\theta} f=\left(-\frac{1}{2} \frac{\mathrm{d}^{2}}{\mathrm{~d} t^{2}}+\frac{1}{2} t^{2}+c \delta\right) f(t),
$$

with $c=\frac{\cot \theta}{\sqrt{2}}$. There is a constant $\gamma>0$ such that if $c>-\gamma$, then $B_{\theta}$ has only positive eigenvalues, if $c=-\gamma$, then 0 is an eigenvalue of $B_{\theta}$, and if $c<\gamma$, then $B_{\theta}$ has exactly one negative eigenvalue. This eigenvalue decreases monotonically to $-\infty$ as $c$ tends to $-\infty$, or equivalently, $\theta$ tends to $\pi$.

Finally, in Section 4 we give an interpretation of the operators $B_{\theta}$ and another selfadjoint extension denoted by $C_{K}$ as operators with a $\delta$ - and $\delta^{\prime}$-interaction at 0 in a Hilbert space $H_{-} \supset L_{2}(\mathbb{R})$. Some bibliographical notes are given in Section 5 .

\section{The Harmonic Oscillator on the Half Line}

First we restrict the harmonic oscillator to the half lines $\mathbb{R}_{ \pm}$. The corresponding minimal operators are given in (6). These operators are in the limit point case at $\pm \infty$ and in the limit circle case at 0 , hence they are not essentially selfadjoint. Their adjoint operators are the ones in (7). Note that for $f \in \mathcal{D}\left(A_{+}^{\max }\right)$ the one-sided limits $f(+0):=\lim _{t \rightarrow 0^{+}} f(t)$ and $f^{\prime}(+0):=\lim _{t \rightarrow 0^{+}} f^{\prime}(t)$ exist. Similarly, for $f \in \mathcal{D}\left(A_{-}^{\max }\right)$ the onesided limits $f(-0):=\lim _{t \rightarrow 0^{-}} f(t)$ and $f^{\prime}(-0):=\lim _{t \rightarrow 0^{-}} f^{\prime}(t)$ exist. 
All selfadjoint extensions of $A_{ \pm}^{\min }$ are given as restrictions of $A_{ \pm}^{\max }$ by appropriate boundary conditions at 0 . In Lemma 2 we will show that exactly one boundary condition is needed.

Recall that the defect index of a closed densely defined linear operator $T$ with respect to $z \in \rho(T)$ is given by

$$
n(T, z):=\operatorname{dim}\left(\operatorname{ker}\left(T^{*}-z\right)\right)
$$

It is well known that the defect indices are constant in the complement of the numerical range

$$
W(T):=\{\langle T x, x\rangle: x \in \mathcal{D}(T),\|x\|=1\},
$$

see, e.g., [9, Ch. V, Theorem 3.2]. It is easy to see that $W(T) \subset \mathbb{R}$ for a symmetric operator $T$. Hence its defect indices are constant in the upper and lower complex plane. Denote them by

$$
n_{+}(T)=\operatorname{dim}\left(\operatorname{ker}\left(T^{*}-z_{+}\right)\right), \quad n_{-}(T)=\operatorname{dim}\left(\operatorname{ker}\left(T^{*}-z_{-}\right)\right),
$$

for any $z_{ \pm} \in \mathbb{C}$ with $\operatorname{Im}\left(z_{ \pm}\right) \in \mathbb{R}_{ \pm}$. By the von Neumann theory, a symmetric operator has selfadjoint extensions if and only if its defect indices are equal (see for instance [14, Ch. 8.2]).

Lemma 1. The defect indices of $A_{ \pm}^{\min }$ are $n_{+}\left(A_{ \pm}^{\min }\right)=n_{-}\left(A_{ \pm}^{\min }\right)=1$ and

$$
W\left(A_{ \pm}^{\min }\right) \subseteq \mathbb{R}_{+}
$$

Proof. We show the lemma only for $A_{+}^{\mathrm{min}}$. For all $f \in \mathcal{D}\left(A_{+}^{\mathrm{min}}\right)$, integration by parts yields

$$
\left\langle A_{+}^{\min } f, f\right\rangle=-\left.f^{\prime}(x) \bar{f}(x)\right|_{0} ^{\infty}+\int_{0}^{\infty}\left|f^{\prime}\right|^{2}+x^{2}|f|^{2} \mathrm{~d} x=\int_{0}^{\infty}\left|f^{\prime}\right|^{2}+x^{2}|f|^{2} \mathrm{~d} x>0
$$

which shows (12). Hence the defect index of $A_{+}^{\min }$ is constant in $\mathbb{C} \backslash \mathbb{R}_{+}$.

It can be easily verified that two pairs of independent solutions of $\left(\mathfrak{A}+\frac{1}{2}\right) f=0$ are

$$
\begin{array}{ll}
\phi_{1}(t)=\mathrm{e}^{\frac{1}{2} t^{2}}, & \phi_{+}(t)=\mathrm{e}^{\frac{1}{2} t^{2}} \int_{t}^{\infty} \mathrm{e}^{-s^{2}} \mathrm{~d} s, \\
\phi_{1}(t)=\mathrm{e}^{\frac{1}{2} t^{2}}, & \phi_{-}(t)=\mathrm{e}^{\frac{1}{2} t^{2}} \int_{-\infty}^{t} \mathrm{e}^{-s^{2}} \mathrm{~d} s=\phi_{+}(-t) .
\end{array}
$$

Observe that $\phi_{+}+\phi_{-}=\sqrt{\pi} \phi_{1}$. Clearly $\left.\phi_{1}\right|_{\mathbb{R}_{+}} \notin L_{2}\left(\mathbb{R}_{+}\right)$, but $\left.\phi_{+}\right|_{\mathbb{R}_{+}} \in L_{2}\left(\mathbb{R}_{+}\right)$. Therefore $\operatorname{ker}\left(A_{+}^{\max }+\frac{1}{2}\right)=\operatorname{span}\left\{\phi_{+} \mid \mathbb{R}_{+}\right\}$and $n_{+}\left(A_{+}^{\min }\right)=n_{-}\left(A_{+}^{\min }\right)=1$.

Analogous calculations show that $\left\langle A_{-}^{\min } f, f\right\rangle \geq 0$ for all $f \in \mathcal{D}\left(A_{-}^{\min }\right),\left.\phi_{1}\right|_{\mathbb{R}_{-}} \notin L_{2}\left(\mathbb{R}_{-}\right)$, $\phi_{-} \mid \mathbb{R}_{-} \in L_{2}\left(\mathbb{R}_{-}\right)$, thus $\operatorname{ker}\left(A_{-}^{\max }+\frac{1}{2}\right)=\operatorname{span}\left\{\phi_{-} \mid \mathbb{R}_{-}\right\}$and $n_{+}\left(A_{-}^{\min }\right)=n_{-}\left(A_{-}^{\min }\right)=1$.

The following result on selfadjoint extensions of $A_{ \pm}^{\text {min }}$ follows easily from the general theory of Sturm - Liouville operators. For the convenience of the reader, we present it here with a proof in order to illustrate the method of indefinite inner product spaces for the description of selfadjoint extensions. 
Lemma 2. All selfadjoint extensions of $A_{ \pm}^{\mathrm{min}}$ are one-dimensional; they are restrictions of $A_{ \pm}^{\max }$ of the form

$$
\mathcal{D}\left(A_{ \pm, \theta}\right)=\left\{f \in \mathcal{D}\left(A_{ \pm}^{\max }\right): \cos (\theta) f_{ \pm}(+0)=\sin (\theta) f_{ \pm}^{\prime}(+0)\right\}
$$

with $\theta \in[0, \pi)$.

Proof. We show the claim only for $A_{+}^{\mathrm{min}}$ since the corresponding assertions for $A_{-}^{\text {min }}$ follow analogously.

By Lemma 1 , the defect index of $A_{ \pm}^{\min }$ is equal to 1 on $\mathbb{C} \backslash \mathbb{R}_{+}$. Two functions $f, g \in \mathcal{D}\left(A_{+}^{\max }\right)$ belong to a particular selfadjoint extension of $A_{+}^{\min }$ if and only if $\left\langle A_{+}^{\max } f, g\right\rangle-\left\langle A_{+}^{\max } f, g\right\rangle=$ 0 . Integration by parts leads to the condition

$$
0=\left\langle A_{+}^{\max } f, g\right\rangle-\left\langle A_{+}^{\max } f, g\right\rangle=f(+0) \bar{g}^{\prime}(+0)-f^{\prime}(+0) \bar{g}(+0) .
$$

On $\mathbb{C}^{2}$ let us define the Hermitian inner product

$$
\left[\left(\begin{array}{l}
x_{1} \\
x_{2}
\end{array}\right),\left(\begin{array}{l}
y_{1} \\
y_{2}
\end{array}\right)\right]=\left\langle\left(\begin{array}{cc}
0 & -\mathrm{i} \\
\mathrm{i} & 0
\end{array}\right)\left(\begin{array}{l}
x_{1} \\
x_{2}
\end{array}\right),\left(\begin{array}{l}
y_{1} \\
y_{2}
\end{array}\right)\right\rangle=\mathrm{i}\left(x_{2} \bar{y}_{1}-x_{1} \bar{y}_{2}\right) .
$$

Then $f, g$ belong to a particular selfadjoint extension of $A_{+}^{\min }$ if and only if $\left(f(+0), f^{\prime}(+0)\right)^{t}$ and $\left(g(+0), g^{\prime}(+0)\right)^{t}$ belong to the same maximal neutral subspace of $\left(\mathbb{C}^{2},[\cdot, \cdot]\right)$. Clearly $e_{+}=(1, \mathrm{i})^{t}$ is a positive and $e_{-}=(-1, \mathrm{i})^{t}$ is a negative vector and $\left\|e_{+}\right\|=\left\|e_{-}\right\|$. Hence all maximal neutral subspaces are given by

$$
L_{\theta}=\operatorname{span}\left\{\left(\begin{array}{c}
1-\mathrm{e}^{-2 \mathrm{i} \theta} \\
\mathrm{i}\left(1+\mathrm{e}^{-2 \mathrm{i} \theta}\right)
\end{array}\right)\right\}=\left(\operatorname{span}\left\{\left(\begin{array}{c}
1+\mathrm{e}^{-2 \mathrm{i} \theta} \\
\mathrm{i}\left(1-\mathrm{e}^{-2 \mathrm{i} \theta}\right)
\end{array}\right)\right\}\right)^{\perp}, \quad \theta \in[0, \pi) .
$$

Therefore all selfadjoint extensions of $A_{+}^{\min }$ are given by

$$
\begin{aligned}
\mathcal{D}\left(A_{+, \theta}\right) & =\left\{f \in \mathcal{D}\left(A_{+}^{\max }\right):\left(\begin{array}{c}
f(+0) \\
f^{\prime}(+0)
\end{array}\right) \in L_{\theta}\right\} \\
& =\left\{f \in \mathcal{D}\left(A_{+}^{\max }\right): f(+0)\left(1+\mathrm{e}^{-2 \mathrm{i} \theta}\right)=-\mathrm{i} f^{\prime}(+0)\left(1-\mathrm{e}^{-2 \mathrm{i} \theta}\right)\right\}
\end{aligned}
$$

with $\theta \in[0, \pi)$. The last description yields (15).

Remark 2. Let $f \in L_{2}\left(\mathbb{R}_{+}\right)$and $g \in L_{2}\left(\mathbb{R}_{-}\right)$such that $g(x)=f(-x)$ for $x \in \mathbb{R}_{-}$. From formula (15) it is clear that $f \in \mathcal{D}\left(A_{+, \theta}\right)$ for some $\theta \in(0, \pi)$ if and only if $g \in \mathcal{D}\left(A_{-, \pi-\theta}\right)$ and $f \in \mathcal{D}\left(A_{+, 0}\right)$ if and only if $g \in \mathcal{D}\left(A_{-, 0}\right)$.

Recall that $\left\{\psi_{n}: n \in \mathbb{N}\right\}$, the set of eigenfunctions of the harmonic oscillator on $\mathbb{R}$ (see $(5)$ ), is an orthonormal basis of $L_{2}(\mathbb{R})$. Denote

$$
\psi_{n,+}=\left.\psi_{n}\right|_{\mathbb{R}_{+}}, \quad \psi_{n,-}=\left.\psi_{n}\right|_{\mathbb{R}_{-}}, \quad n \in \mathbb{N}_{0}
$$

Clearly both $\left\{\psi_{2 n, \pm}: n \in \mathbb{N}_{0}\right\}$ and $\left\{\psi_{2 n+1, \pm}: n \in \mathbb{N}_{0}\right\}$ form a complete orthogonal systems on $\mathbb{R}_{ \pm}$. With these observations we can calculate the spectrum of the operators $A_{ \pm, \theta}$ for $\theta=0$ and $\theta=\frac{\pi}{2}$.

Corollary 1. Let $A_{ \pm, \theta}$ be as in (15). 
1. $\sigma\left(A_{ \pm, 0}\right)=\sigma_{p}\left(A_{ \pm, 0}\right)=\left\{2 n+\frac{3}{2}: n \in \mathbb{N}_{0}\right\}$ and the corresponding eigenfunctions are $\psi_{2 n+1, \pm}, n \in \mathbb{N}_{0}$.

2. $\sigma\left(A_{ \pm, \frac{\pi}{2}}\right)=\sigma_{p}\left(A_{ \pm, \frac{\pi}{2}}\right)=\left\{2 n+\frac{1}{2}: n \in \mathbb{N}_{0}\right\}$ and the corresponding eigenfunctions are $\psi_{2 n, \pm}, n \in \mathbb{N}_{0}$.

Proof. We will prove the claim only for $A_{+, 0}$. All other statements are proved analogously. Since all $\psi_{2 n+1}$ are odd functions, it follows that $\psi_{2 n+1}(0)=0$ and therefore their restrictions $\psi_{2 n+1,+}$ belong to $\mathcal{D}\left(A_{+, 0}\right)$. Moreover, $A_{+, 0} \psi_{2 n+1,+}=\left(2 n+\frac{3}{2}\right) \psi_{2 n+1,+}$, hence $\left\{2 n+\frac{3}{2}: n \in \mathbb{N}_{0}\right\} \subset \sigma_{p}\left(A_{ \pm, 0}\right)$. Now the claim follows from the completeness of the system $\left\{\psi_{2 n+1,+}\right\}$ in $L_{2}\left(\mathbb{R}_{+}\right)$.

From the asymptotic expansion (1) of solutions of the equation $\mathfrak{A} y=\lambda y$ it is clear that for every $\lambda \in \mathbb{R}$ there is exactly one solution $y_{\lambda}$ which is square integrable on $\mathbb{R}_{+}$. It belongs to the domain of exactly one selfadjoint extension $A_{+, \theta}$, namely the one with $\theta \in[0, \pi)$ such that $\cos \theta y_{\lambda}(0)=\sin \theta y_{\lambda}^{\prime}(0)$. The expansion (1) also shows that all eigenvalues are simple.

Since $W\left(A_{ \pm}^{\min }\right) \subseteq(0, \infty)$, the non-positive spectrum $\sigma\left(A_{\theta}\right) \cap(-\infty, 0]$ of any selfadjoint extension $A_{\theta}$ consists of at most one eigenvalue of multiplicity at most 1, see [14, Ch. 8.4, Cor. 2]. The lemma below deals with these eigenvalues, but as a first step we need to define some functions.

Let $\omega \geq 0$. On $[0, \infty)$ consider the Cauchy problem

$$
\begin{gathered}
\left(-\frac{1}{2} \frac{\mathrm{d}^{2}}{\mathrm{~d} t^{2}}+\frac{1}{2} t^{2}\right) u(t, \omega)=-\omega^{2} u(t, \omega), \\
u(0, \omega)=1, \quad u_{t}^{\prime}(0, \omega)=0 .
\end{gathered}
$$

Let us write $u(t, \omega)$ as power series

$$
u(t, \omega)=\sum_{n=0}^{\infty} a_{n}(\omega) t^{n}
$$

Replacing (20) in (18) we obtain $a_{2 n+1}(\omega)=0$ and

$$
\begin{aligned}
a_{0}(\omega) & =1, \quad a_{2}(\omega)=\omega^{2} \\
a_{2 n+2}(\omega) & =\frac{1}{(2 n+2)(2 n+1)}\left(2 \omega^{2} a_{2 n}(\omega)+a_{2 n-2}(\omega)\right), \quad n \geq 1 .
\end{aligned}
$$

Let us show that the series converges for all $t>0$. To this end we will show that for every $\omega \geq 0$ there is a constant $q(\omega)$ such that

$$
a_{2 n}(\omega) \leq \frac{q(\omega)}{n !}, \quad n \in \mathbb{N}
$$

Assume that this inequality is true for numbers $n-1$ and $n$. Then, due to (21), we have

$$
\begin{aligned}
a_{2 n+2} & \leq \frac{1}{(2 n+2)(2 n+1)}\left(\frac{2 \omega^{2} q(\omega)}{n !}+\frac{q(\omega)}{(n-1) !}\right) \\
& =\frac{q(\omega)}{(n+1) !}\left(\frac{\omega^{2}}{2 n+1}+\frac{n}{2(2 n+1)}\right) .
\end{aligned}
$$


Since $\frac{\omega^{2}}{2 n+1}+\frac{n}{2(2 n+1)} \rightarrow \frac{1}{4}$ for $n \rightarrow \infty$, there is a natural number $n_{0}(\omega)$ such that $\frac{\omega^{2}}{2 n+1}+$ $\frac{n}{2(2 n+1)}<1$ for every $n \geq n_{0}(\omega)$. Thus, (22) holds if we take

$$
q(\omega)=\max \left\{1,2 ! \cdot a_{2}(\omega), \ldots, n_{0} ! \cdot a_{2 n_{0}}(\omega)\right\} .
$$

Due to $(22)$ the series (20) converges and $u(t, \omega) \leq q(\omega) \mathrm{e}^{t^{2}}$ for $\omega \geq 0, t>0$.

Note that all $a_{2 n}(\omega)$ are positive increasing functions of $\omega$, so for every $t>0, u(t, \omega)$ is an increasing function with respect to $\omega$ and for every $\omega \geq 0, u(t, \omega)$ is a positive increasing function with respect to $t$, so $u(t, \omega) \notin L_{2}\left(\mathbb{R}_{+}\right)$. For the special case $\omega=0$ we obtain

$$
u(t, 0)=1+\sum_{n=1}^{\infty} \frac{t^{4 n}}{\prod_{k=1}^{n} 4 k(4 k-1)} .
$$

Due to the inequalities

$$
\frac{1}{3^{2} 2 n(2 n-1)}<\frac{1}{4 n(4 n-1)}<\frac{1}{2^{2} 2 n(2 n-1)}, \quad n \in \mathbb{N},
$$

we have

$$
\cosh \left(\frac{x^{2}}{3}\right)<u(t, 0)<\cosh \left(\frac{x^{2}}{2}\right), \quad t>0 .
$$

Now let us define (compare with (13))

$$
v(t, \omega):=u(t, \omega) \int_{t}^{\infty}(u(s, \omega))^{-2} \mathrm{~d} s
$$

and

$$
G(\omega):=v(0, \omega)=\int_{0}^{\infty}(u(s, \omega))^{-2} \mathrm{~d} s .
$$

Lemma 3. Let $A_{ \pm, \theta}$ be as in (15) and let $\alpha_{A}=\arctan (G(0))$.

1. $A_{+, \theta}$ has the eigenvalue 0 if and only if $\theta=\pi-\alpha_{A}$. It has a negative eigenvalue if and only if $\theta \in\left(\pi-\alpha_{A}, \pi\right)$. Moreover, let $\lambda_{j}$ be eigenvalues of $A_{+, \theta_{j}}$ for $j=1,2$. If $\lambda_{2}<\lambda_{1}<0$, then $\pi-\alpha_{A}<\theta_{1}<\theta_{2}<\pi$.

2. $A_{-, \theta}$ has the eigenvalue 0 if and only if $\theta=\alpha_{A}$. It has a negative eigenvalue if and only if $\theta \in\left(0, \alpha_{A}\right)$. Moreover, let $\lambda_{j}$ be eigenvalues of $A_{-, \theta_{j}}$ for $j=1,2$. If $\lambda_{2}<\lambda_{1}<0$, then $\alpha_{A}>\theta_{1}>\theta_{2}>0$.

Proof. We proof only the item 1. The claims in the item 2 follow from the item 1 and Remark 2. Since $u(t, \omega)$ is positive and increasing both in $t$ and $\omega$, it follows from (24) that

$$
\begin{aligned}
v(t, \omega) & <u(t, \omega) \int_{t}^{\infty}(u(t, \omega))^{-1}(u(s, \omega))^{-1} \mathrm{~d} s=\int_{t}^{\infty}(u(s, \omega))^{-1} \mathrm{~d} s \\
& \leq \int_{t}^{\infty}(u(s, 0))^{-1} \mathrm{~d} s<\int_{t}^{\infty}\left(\cosh \left(s^{2} / 3\right)\right)^{-1} \mathrm{~d} s \in L_{2}\left(\mathbb{R}_{+}\right) .
\end{aligned}
$$


It is easy to check that $v(\cdot, \omega)$ satisfies $(18)$ and

$$
v_{t}^{\prime}(t, \omega)=-\frac{1}{u(t, \omega)}+u^{\prime}(t, \omega) \int_{t}^{\infty}(u(s, \omega))^{-2} \mathrm{~d} s,
$$

therefore, by $(19), v_{t}^{\prime}(0, \omega)=-1$. It follows from (26) that

$$
v_{t}^{\prime}(0, \omega)=-\frac{v(0, \omega)}{G(\omega)}
$$

which is equivalent to the boundary condition (15) with $\theta$ such that $\tan \theta=-G(\omega)$, that is, $\theta=-\arctan (G(\omega)) \in(\pi / 2, \pi)$. Observe that $G(\omega)$ is decreasing and continuous in $\omega$ and $\lim _{\omega \rightarrow \infty} G(\omega)=0$. Hence $\theta$ is increasing in $\omega$ and tends to $\pi$ as $\omega \rightarrow \infty$. For the special case $\omega=0$ we obtain $\theta=\pi / 2-\alpha_{A}$ where $\alpha_{A}=\arctan (G(0))$.

\section{One-Dimensional Restriction of the Harmonic Oscillator and Classification of All Selfadjoint Extensions}

In this section we consider the harmonic oscillator $B$ on the real line defined by (9). The operator $B$ is closed and symmetric, but not selfadjoint. Let us recall also the definition (8) of $A_{0}$.

Remark 3. The domain of $A_{0}$ can be viewed as $\mathcal{D}\left(A_{0}\right)=\mathcal{D}\left(A_{-}^{\mathrm{min}}\right) \oplus \mathcal{D}\left(A_{+}^{\mathrm{min}}\right)$, hence it is easy to see that its adjoint is given by

$$
\begin{aligned}
& A_{0}^{*} f(t)= \begin{cases}A_{+}^{\max } f_{+}(t), & t>0, \\
A_{-}^{\max } f_{-}(t), & t<0,\end{cases} \\
& \mathcal{D}\left(A_{0}^{*}\right)=\left\{f \in L_{2}(\mathbb{R}): f_{ \pm} \in \mathcal{D}\left(A_{ \pm}^{\max }\right)\right\}=\mathcal{D}\left(A_{-}^{\max }\right) \oplus \mathcal{D}\left(A_{+}^{\max }\right) .
\end{aligned}
$$

It should be noted that $A_{0}^{*}$ and the selfadjoint extensions $B_{\theta}$ and $C_{K}$ of $B$ and $C$ which will be calculated below are not differential operators on $L_{2}(\mathbb{R})$ in the classical sense because functions in their domains need not be continuous or differentiable in 0 .

Lemma 4. We have the chain of extensions $A_{0} \subset B \subset B^{*} \subset A_{0}^{*}$ and

$$
\begin{aligned}
& B^{*} f(t)=A_{0}^{*} f(t), \\
& \mathcal{D}\left(B^{*}\right)=\left\{f: \mathbb{R} \rightarrow \mathbb{C}: f_{ \pm} \in \mathcal{D}\left(A_{ \pm}^{\max }\right), f(-0)=f(+0)\right\} .
\end{aligned}
$$

Proof. Note that $A_{0} \subset B$, hence $B^{*} \subset A_{0}^{*}$. Let $f \in \mathcal{D}(B)$ and $g \in \mathcal{D}\left(A_{0}^{*}\right)$. Then $f$ and $f^{\prime}$ are continuous in $0, f(0)=0$ and $g_{ \pm} \in \mathcal{D}\left(A_{ \pm}^{\max }\right)$. Hence integration by parts yields

$$
\begin{aligned}
\langle B f, g\rangle-\left\langle f, A_{0}^{*} g\right\rangle & =-\int_{-\infty}^{0} f^{\prime \prime} \bar{g} \mathrm{~d} t-\int_{0}^{\infty} f^{\prime \prime} \bar{g} \mathrm{~d} t+\int_{-\infty}^{0} f \bar{g}^{\prime \prime} \mathrm{d} t+\int_{0}^{\infty} f \bar{g}^{\prime \prime} \mathrm{d} t \\
& =f^{\prime}(0)(\bar{g}(+0)-\bar{g}(-0)) .
\end{aligned}
$$

Therefore $g \in \mathcal{D}\left(B^{*}\right)$ if and only if $g$ is continuous in 0 and in this case $B^{*} g=A_{0}^{*} g$. 
Note that functions in the domain of $B^{*}$ are continuous but their derivative may have a discontinuity in 0 .

Analogously to Lemma 2 we now classify all selfadjoint extensions of $B$.

Proposition 1. The defect indices of $B$ are $n_{+}(B)=n_{-}(B)=1$. Hence all selfadjoint extensions of $B$ are one-dimensional restrictions of $B^{*}$. They are given by (11).

Proof. Observe that $B$ is a restriction of $A$, hence for the numerical ranges we have the inclusion $W(B) \subset W(A) \subset[0, \infty)$, so the defect index of $B$ is constant in $\mathbb{C} \backslash[0, \infty)$ and it suffices to show that $\operatorname{dim}\left(\operatorname{ker}\left(B^{*}+\frac{1}{2}\right)\right)=1$.

The proof of Lemma 1 shows that every $L_{2}$-solution of $\left(\mathfrak{A}+\frac{1}{2}\right) f=0$ must be of the form

$$
f=\alpha_{-} \phi_{-} \chi_{(-\infty, 0)}+\alpha_{+} \phi_{+} \chi_{(0,+\infty)}
$$

with $\alpha_{ \pm} \in \mathbb{C}$ and $\phi_{ \pm}$as in (13) and (14). Here $\chi_{(-\infty, 0)}$ and $\chi_{(0,+\infty)}$ are the indicator functions of the sets $(-\infty, 0)$ and $(0,+\infty)$ respectively. Clearly every function (28) belongs to $\mathcal{D}\left(A_{0}^{*}\right)$ and $f(-0)=\frac{\alpha_{-}}{2} \sqrt{\pi}$ and $f(+0)=\frac{\alpha_{+}}{2} \sqrt{\pi}$. For $f \in \mathcal{D}\left(B^{*}\right)$ we must have that $f(-0)=f(+0)$. Therefore $\operatorname{ker}\left(B^{*}+\frac{1}{2}\right)=\operatorname{span}\left\{\phi_{-} \chi_{(-\infty, 0)}+\phi_{+} \chi_{(0,+\infty)}\right\}$. Thus $n\left(B,-\frac{1}{2}\right)=$ 1.

Let us now determine all selfadjoint extensions of $B$. This is equivalent to determine all selfadjoint restrictions of $B^{*}$. Let $f, g \in \mathcal{D}\left(B^{*}\right)$. Performing integration by parts we find

$$
\left\langle f, B^{*} g\right\rangle-\left\langle B^{*} f, g\right\rangle=\left[f^{\prime}(-0)-f^{\prime}(+0)\right] \bar{g}(0)-\left[\bar{g}^{\prime}(-0)-\bar{g}^{\prime}(+0)\right] f(0) .
$$

Set

$$
G=\left(\begin{array}{ccc}
0 & -\mathrm{i} & \mathrm{i} \\
\mathrm{i} & 0 & 0 \\
-\mathrm{i} & 0 & 0
\end{array}\right)
$$

Then $f, g$ belong to a particular selfadjoint extension of $B$ if and only if $\left(f(0), f^{\prime}(-0), f^{\prime}(+0)\right)^{t}$ and $\left(g(0), g^{\prime}(-0), g^{\prime}(+0)\right)^{t}$ belong to a maximal neutral subspace of $\left(\mathbb{C}^{3},[\cdot, \cdot]\right)$ where

$$
\left[\left(\begin{array}{l}
x_{1} \\
x_{2} \\
x_{3}
\end{array}\right),\left(\begin{array}{l}
y_{1} \\
y_{2} \\
y_{3}
\end{array}\right)\right]:=\left\langle G\left(\begin{array}{l}
x_{1} \\
x_{2} \\
x_{3}
\end{array}\right),\left(\begin{array}{l}
y_{1} \\
y_{2} \\
y_{3}
\end{array}\right)\right\rangle=\mathrm{i}\left[x_{1}\left(\bar{y}_{3}-\bar{y}_{2}\right)+\bar{y}_{1}\left(x_{2}-x_{3}\right)\right] .
$$

The eigenvalues of $G$ are 0 and $\pm \sqrt{2}$ with eigenspaces

$$
\begin{aligned}
\operatorname{ker} G & =\operatorname{span}\left\{(0,1,1)^{t}\right\}, \\
L_{+}:=\operatorname{ker}(G-\sqrt{2}) & =\operatorname{span}\left\{(\mathrm{i} \sqrt{2},-1,1)^{t}\right\}, \\
L_{-}:=\operatorname{ker}(G+\sqrt{2}) & =\operatorname{span}\left\{(-\mathrm{i} \sqrt{2},-1,1)^{t}\right\} .
\end{aligned}
$$

Note that $L_{ \pm}$are maximal positive and maximal negative subspaces of $\mathbb{C}^{3}$ respectively. Hence any maximal neutral subspace of $\mathbb{C}^{3}$ has dimension 2. They are of the form $\operatorname{ker} G \oplus$ $\{v+K v: v \in \operatorname{ker}(G-\sqrt{2})\}$ where $K$ is an isometry from $L_{+}$to $L_{-}$. Clearly all such 
isometries are of the form $v_{+} \mapsto \mathrm{e}^{-2 \mathrm{i} \theta} v_{-}$where $v_{ \pm} \in L_{ \pm}$. In summary, all maximal neutral subspaces are

$$
L_{\theta}=\operatorname{span}\left\{\left(\begin{array}{l}
0 \\
1 \\
1
\end{array}\right),\left(\begin{array}{c}
\mathrm{i} \sqrt{2} \\
-1 \\
1
\end{array}\right)+\mathrm{e}^{2 \mathrm{i} \theta}\left(\begin{array}{c}
-\mathrm{i} \sqrt{2} \\
-1 \\
1
\end{array}\right)\right\}, \quad \theta \in[0, \pi) .
$$

This can be rewritten as

$$
L_{\theta}=\operatorname{span}\left\{\left(\begin{array}{l}
0 \\
1 \\
1
\end{array}\right),\left(\begin{array}{c}
\sqrt{2} \sin (\theta) \\
-\cos (\theta) \\
\cos (\theta)
\end{array}\right)\right\}=\left(\operatorname{span}\left\{\left(\begin{array}{c}
\sqrt{2} \cos (\theta) \\
\sin (\theta) \\
-\sin (\theta)
\end{array}\right)\right\}\right)^{\perp} .
$$

It follows that $f \in \mathcal{D}\left(B_{\theta}\right)$ if and only if $f \in \mathcal{D}\left(B^{*}\right)$ and

$$
\sqrt{2} \cos \theta f(0)+\sin \theta\left[f^{\prime}(-0)-f^{\prime}(+0)\right]=0 .
$$

\section{Remark 4.}

1. Observe that functions $f$ in $\mathcal{D}(B)^{*}$ which satisfy $\left\{\left(f(0), f^{\prime}(-0), f^{\prime}(+0)\right\} \in \operatorname{ker} G\right.$ belong to $\mathcal{D}(B)$.

2. The selfadjoint extensions of $B$ can be divided into the following cases:

(a) $\theta=\frac{\pi}{2}$. In this case the boundary condition (29) is simplified to

$$
f^{\prime}(-0)-f^{\prime}(+0)=0
$$

That is, $f$ and $f^{\prime}$ are continuous and we obtain the classical harmonic oscillator: $B_{\pi / 2}=A$.

(b) $\theta=0$. In this case the boundary condition (29) is simplified to $f(0)=0$.

(c) $\theta \in(0, \pi) \backslash\{\pi / 2\}$. The boundary condition (29) can be written as

$$
f(0)=\frac{\tan (\theta)}{\sqrt{2}}\left[f^{\prime}(+0)-f^{\prime}(-0)\right] .
$$

Hence any function in $\mathcal{D}\left(B_{\theta}\right)$ is continuous but its derivative has a jump in $t=0$ which is proportional to the value of $f$ in 0 . Two different selfadjoint extensions of $B$ have different constants of proportionality.

3. For every $n \in \mathbb{N}_{0}$, the function $\psi_{2 n+1}$ from (5) is an eigenfunction of $B_{\theta}$ with eigenvalue $2 n+3 / 2$. So the odd eigenvalues of the harmonic oscillator are not affected by the boundary condition at 0 .

An interpretation of these operators as a differential operator with a $\delta$-interaction at 0 is given in Section 4 .

Let $\lambda \in \mathbb{C}$. By the asymptotic expansion (1) the equation $\mathfrak{A} y=\lambda y$ has square integrable solutions $y_{ \pm}$on $\mathbb{R}_{ \pm}$which are unique up to a constant factor. Let us define

$$
y(x)= \begin{cases}y_{+}(x), & x \geq 0 \\ y_{+}(-x), & x<0\end{cases}
$$


Then clearly $y \in \mathcal{D}\left(B^{*}\right)$ and it is, up to a constant factor, the unique solution of $\left(B^{*}-\lambda\right) y=$ 0 . Moreover, $y \in \mathcal{D}\left(B_{\theta}\right)$ where $\theta$ is the unique number in $[0, \pi)$ such that $\sqrt{2} \cos \theta f(0)=$ $\sin \theta\left[f^{\prime}(+0)-f^{\prime}(-0)\right]$.

This shows that, as in the case of selfadjoint extensions of $A_{0}$, every $\lambda \in \mathbb{R}$ appears as eigenvalue of exactly one selfadjoint extension of $B$ and that every eigenvalue is simple. Moreover, any given $B_{\theta}$ can have at most one negative eigenvalue.

As in Lemma 3 we can identify all $\theta$ such that $B_{\theta}$ has a negative eigenvalue.

Lemma 5. Let $B_{\theta}$ be as in Proposition 1 and let $\alpha_{B}=\arctan \left(\frac{G(0)}{\sqrt{2}}\right)$ with $G$ as in $(26)$. Then $B_{\theta}$ has the eigenvalue 0 if and only if $\theta=\pi-\alpha_{B}$. It has a negative eigenvalue if and only if $\theta \in\left(\pi-\alpha_{B}, \pi\right)$. The corresponding eigenvalue $\lambda=-\omega^{2}$ can be found via the equality $\tan (\theta)=-\frac{G(\omega)}{\sqrt{2}}$. Moreover, let $\lambda_{j}$ be eigenvalues of $B_{\theta_{j}}$ for $j=1$, 2. If $\lambda_{2}<\lambda_{1}<0$, then $\pi-\alpha_{B}<\theta_{1}<\theta_{2}<\pi$.

Proof. Let $\lambda=-\omega^{2}<0$ and $y$ be as in $(30)$ with $y_{+}=v(\cdot, \omega)$ (see (25)). Then $y$ is an eigenfunction of $B_{\theta}$ with eigenvalue $\lambda$ if and only if $\tan (\theta)=-\frac{G(\omega)}{\sqrt{2}}$. Hence negative eigenvalues occur if and only if $\theta \in\left(\pi-\arctan \left(\frac{G(0)}{\sqrt{2}}\right), \pi\right)$. Since $G$ is decreasing in $\omega$ with $\lim _{\omega \rightarrow \infty} G(\omega)=0$, also the last claim follows.

For $\lambda \in-\left(2 \mathbb{N}-\frac{1}{2}\right)$ we can calculate the corresponding eigenfunctions by a recursion formula.

Lemma 6. Let $n \in \mathbb{N}$ and $\phi_{ \pm}$be as in (13) and (14). Set $\phi_{ \pm, n}(t):=\left(\frac{\mathrm{d}}{\mathrm{d} t}+t\right)^{n} \phi_{ \pm}(t)$ for $t \in \mathbb{R}_{ \pm}$and

$$
u_{n}(t):= \begin{cases}\phi_{+, n}(t), & t>0 \\ \phi_{-, n}(t), & t<0 .\end{cases}
$$

Then $u_{2 n} \in \mathcal{D}\left(B^{*}\right)$. That is, $u_{2 n}$ defines a selfadjoint extension $B_{\theta}$ of $B$ and it is an eigenfunction of $B_{\theta}$ with eigenvalue $-2 n-\frac{1}{2}$.

Proof. Clearly $u_{n} \in \mathcal{D}\left(A_{0}^{\max }\right)$. It is easy to check that $\phi_{+}(t)=\phi_{-}(-t)$ for $t>0$. Moreover, a straightforward calculation shows

$$
\left(\mathfrak{A}+n+\frac{1}{2}\right) \phi_{ \pm, n}=0 .
$$

Hence $\phi_{+, n}(t)=(-1)^{n} \phi_{-, n}(-t)$ for $t>0$. So $u_{2 n}$ is continuous in 0 , and therefore it belongs to $\mathcal{D}\left(B^{*}\right)$. If in addition we had $u_{2 n}^{\prime}(-0)=u_{2 n}^{\prime}(+0)$, then $u_{2 n} \in \mathcal{D}(A)$, and $-2 n-\frac{1}{2}$ would be an eigenvalue of $A$, in contradiction to (4).

\section{Two-Dimensional Restriction of the Harmonic Oscillator and Classification of Its Selfadjoint Extensions}

Let us further restrict the harmonic oscillator on the real line. We consider the following restriction $C$ of the selfadjoint operator $A$ :

$$
C f:=A f, \quad \mathcal{D}(C):=\left\{f \in \mathcal{D}(A): f(0)=f^{\prime}(0)=0\right\} .
$$


The operator $C$ is closed and symmetric, but not selfadjoint. It is easy to see that

$$
C^{*}=A_{0}^{*}
$$

The operator $C$ is closely related to the harmonic oscillator on the half lines $\mathbb{R}_{ \pm}$because

$$
C=\left(C^{*}\right)^{*}=\left(A_{0}^{*}\right)^{*}=\overline{A_{0}}=\overline{A_{-}^{\min }} \oplus \overline{A_{+}^{\min }} .
$$

Analogously to Lemma 2 and Proposition 1 we now classify all selfadjoint extensions of $C$. Observe that the selfadjoint extensions of $C$ are exactly those of $A_{0}$.

Recall that $U(2)$ is the set of all unitary $2 \times 2$ matrices.

Proposition 2. The defect indices $n_{+}(C)$ and $n_{-}(C)$ of $C$ are such that $n_{+}(C)=$ $n_{-}(C)=2$. Hence all selfadjoint extensions of $C$ are two-dimensional restrictions of $C^{*}$. There is a bijection from $U(2)$ to the set of all selfadjoint extensions of $C$ given as follows: for every $K=\left(k_{l m}\right)_{l, m=1}^{2} \in U(2)$, the operator

$$
\begin{aligned}
& C_{K} f=\mathfrak{A} f, \\
& \mathcal{D}\left(C_{K}\right)=\left\{\begin{array}{c}
0= \\
f \in \mathcal{D}\left(C^{*}\right): \quad\left(1-k_{11}\right) f(-0)+\mathrm{i}\left(1+k_{11}\right) f^{\prime}(-0) \\
\quad+\mathrm{i} k_{12} f(+0)-k_{12} f^{\prime}(+0), \\
0=-k_{21} f(-0)+\mathrm{i} k_{21} f^{\prime}(-0) \\
+\mathrm{i}\left(1+k_{22}\right) f(+0)+\left(1-k_{22}\right) f^{\prime}(+0)
\end{array}\right\}
\end{aligned}
$$

is a selfadjoint extension of $C$. There are no other selfadjoint extensions and $C_{K}=C_{\widetilde{K}}$ if and only if $K=\widetilde{K}$.

For a parametrization of the selfadjoint extensions with four real parameters, see the corollary after the proof of this proposition.

Proof. From Lemma 2 we know that $n_{+}\left(A_{ \pm}^{\min }\right)=n_{-}\left(A_{ \pm}^{\min }\right)=1$, so

$$
\operatorname{dim}\left(\operatorname{ker}\left(A_{ \pm}^{\max }-\mathrm{i}\right)\right)=\operatorname{dim}\left(\operatorname{ker}\left(A_{ \pm}^{\max }+\mathrm{i}\right)\right)=1 .
$$

Hence there are functions $\psi_{ \pm} \neq 0$ such that $\operatorname{ker}\left(A_{ \pm}^{\max }-\mathrm{i}\right)=\operatorname{span}\left\{\psi_{ \pm}\right\}$. From Remark 3 we have $\eta \in \operatorname{ker}\left(A_{0}^{*}-\mathrm{i}\right)$ if and only if $\left.\eta\right|_{\mathbb{R}_{ \pm}} \in \operatorname{ker}\left(A_{ \pm}^{\max }-\mathrm{i}\right)$. Therefore (compare with (28))

$$
\operatorname{ker}\left(A_{0}^{*}-\mathrm{i}\right)=\operatorname{span}\left\{\chi_{(-\infty, 0)} \psi_{-}, \chi_{(0,+\infty)} \psi_{+}\right\}
$$

and $n_{+}\left(A_{0}^{*}\right)=2$. Analogously $n_{-}\left(A_{0}^{*}\right)=2$ can be shown.

Now let us determine all selfadjoint extensions of $A_{0}$ which is equivalent to determine all selfadjoint restrictions of $A_{0}^{*}$. Again we apply integration by parts and find

$$
\left\langle f, A_{0}^{*} g\right\rangle-\left\langle A_{0}^{*} f, g\right\rangle=f(+0) \bar{g}^{\prime}(+0)-f^{\prime}(+0) \bar{g}(+0)-f(-0) \bar{g}^{\prime}(-0)+f^{\prime}(-0) \bar{g}(-0)
$$

for all $f, g \in \mathcal{D}\left(A_{0}^{*}\right)$.

Hence $f, g$ belong to a particular selfadjoint extension of $A_{0}$ if and only if $\left(f(-0), f^{\prime}(-0), f(+0), f^{\prime}(+0)\right)^{t}$ and $\left(g(-0), g^{\prime}(-0), g(+0), g^{\prime}(+0)\right)^{t}$ belong to a maximal 
neutral subspace of $\left(\mathbb{C}^{4},[\cdot, \cdot]\right)$ where

$$
\begin{aligned}
& {\left[\left(\begin{array}{l}
x_{1} \\
x_{2} \\
x_{3} \\
x_{4}
\end{array}\right),\left(\begin{array}{l}
y_{1} \\
y_{2} \\
y_{3} \\
y_{4}
\end{array}\right)\right]:=\left\langle\left(\begin{array}{cccc}
0 & -\mathrm{i} & 0 & 0 \\
\mathrm{i} & 0 & 0 & 0 \\
0 & 0 & 0 & \mathrm{i} \\
0 & 0 & -\mathrm{i} & 0
\end{array}\right)\left(\begin{array}{l}
x_{1} \\
x_{2} \\
x_{3} \\
x_{4}
\end{array}\right),\left(\begin{array}{l}
y_{1} \\
y_{2} \\
y_{3} \\
y_{4}
\end{array}\right)\right\rangle } \\
&= \mathrm{i}\left(x_{1} \bar{y}_{2}-x_{2} \bar{y}_{1}-x_{3} \bar{y}_{4}+x_{4} \bar{y}_{3}\right) .
\end{aligned}
$$

Every maximal neutral subspace has dimension 2. Let

$$
\begin{aligned}
v_{1} & =\frac{1}{\sqrt{2}}(1, \mathrm{i}, 0,0)^{t}, \quad v_{2}=\frac{1}{\sqrt{2}}(0,0, \mathrm{i}, 1)^{t}, \\
w_{1} & =\frac{1}{\sqrt{2}}(1,-\mathrm{i}, 0,0)^{t}, w_{2}=\frac{1}{\sqrt{2}}(0,0,-\mathrm{i}, 1)^{t} .
\end{aligned}
$$

Then $L_{+}=\operatorname{span}\left\{v_{1}, v_{2}\right\}$ is a maximal positive and $L_{-}=\operatorname{span}\left\{w_{1}, w_{2}\right\}$ is a maximal negative subspace of $\left(\mathbb{C}^{4},[\cdot, \cdot]\right)$ and all maximal neutral subspaces are of the form

$$
L_{K}=\left\{v+K v: v \in L_{+}\right\}=\left\{w+K^{*} w: w \in L_{-}\right\}^{[\perp]}=\left\{w-K^{*} w: w \in L_{-}\right\}^{\perp}
$$

where $K$ is a unitary operator from $L_{+}$to $L_{-}$and $[\perp]$ denotes the orthogonal complement with respect to the inner product $[\cdot, \cdot]$. With respect to the basis vectors $v_{1}, v_{2}, w_{1}, w_{2}, K$ can be written as quadratic matrix

$$
K=\left(\begin{array}{ll}
k_{11} & k_{12} \\
k_{21} & k_{22}
\end{array}\right)
$$

with $k_{j k} \in \mathbb{C}$ (for the form of these numbers see the corollary after this proof). With respect to the standard unit vectors $\mathrm{e}_{1}, \mathrm{e}_{2}, \mathrm{e}_{3}, \mathrm{e}_{4}$ in $\mathbb{C}^{4}$, the space $L_{K}$ can be written as

$$
\begin{aligned}
L_{K} & =\operatorname{span}\left\{\left(\begin{array}{c}
1+k_{11} \\
\mathrm{i}\left(1-k_{11}\right) \\
-\mathrm{i} k_{21} \\
k_{21}
\end{array}\right), \quad\left(\begin{array}{c}
k_{12} \\
-\mathrm{i} k_{12} \\
\mathrm{i}\left(1-k_{22}\right) \\
1+k_{22}
\end{array}\right)\right\} \\
& \left.=\operatorname{span}\left\{\left(\begin{array}{c}
1-\bar{k}_{11} \\
-\mathrm{i}\left(1+\bar{k}_{11}\right) \\
-\mathrm{i} \bar{k}_{12} \\
-\bar{k}_{12}
\end{array}\right),\left(\begin{array}{c}
-\bar{k}_{21} \\
-\mathrm{i} \bar{k}_{21} \\
-\mathrm{i}\left(1+\bar{k}_{22}\right) \\
1-\bar{k}_{22}
\end{array}\right)\right\}\right)^{\perp}
\end{aligned}
$$

where $K=\left(k_{i j}\right)_{i j=1}^{2}$ as in (33). From (34) it follows that every selfadjoint extension of $C$ is given by $(32)$.

It is well-known that $U(2)$ is parameterized by four real parameters $\phi, \alpha, \beta_{1}, \beta_{2} \in \mathbb{R}$ : Every $K \in U(2)$ is given by

for fixed $\phi, \alpha, \beta_{1}, \beta_{2}$.

$$
K=\mathrm{e}^{\mathrm{i} \phi}\left(\begin{array}{cc}
\mathrm{e}^{\mathrm{i} \beta_{1}} \sin \alpha & \mathrm{e}^{-\mathrm{i} \beta_{2}} \cos \alpha \\
\mathrm{e}^{\mathrm{i} \beta_{2}} \cos \alpha & -\mathrm{e}^{-\mathrm{i} \beta_{1}} \sin \alpha
\end{array}\right)
$$


Therefore the boundary conditions in (32) can be rewritten as follows:

Corollary 2. Let $K \in U(2)$ be as in (35). Then $f \in \mathcal{D}\left(C_{K}\right)$ if and only if $f \in \mathcal{D}\left(C^{*}\right)$ and $f$ satisfies the boundary conditions

$$
\begin{aligned}
& 0=\left(1-\mathrm{e}^{\mathrm{i} \phi} \mathrm{e}^{\mathrm{i} \beta_{1}} \sin \alpha\right) f(-0)+\mathrm{i}\left(1+\mathrm{e}^{\mathrm{i} \phi} \mathrm{e}^{\mathrm{i} \beta_{1}} \sin \alpha\right) f^{\prime}(-0) \\
& +\quad \mathrm{ie}^{\mathrm{i} \phi} \mathrm{e}^{-\mathrm{i} \beta_{2}} \cos \alpha f(+0)-\quad \mathrm{e}^{\mathrm{i} \phi} \mathrm{e}^{-\mathrm{i} \beta_{2}} \cos \alpha f^{\prime}(+0), \\
& 0=\quad-\mathrm{e}^{\mathrm{i} \phi} \mathrm{e}^{\mathrm{i} \beta_{2}} \cos \alpha f(-0)+\quad \mathrm{ie}^{\mathrm{i} \phi} \mathrm{e}^{\mathrm{i} \beta_{2}} \cos \alpha f^{\prime}(-0) \\
& +\mathrm{i}\left(1-\mathrm{e}^{\mathrm{i} \phi} \mathrm{e}^{-\mathrm{i} \beta_{1}} \sin \alpha\right) f(+0)+\left(1+\mathrm{e}^{\mathrm{i} \phi} \mathrm{e}^{-\mathrm{i} \beta_{1}} \sin \alpha\right) f^{\prime}(+0) \text {. }
\end{aligned}
$$

In the following subsections we discuss particular choices of $K$.

\subsection{Classical Harmonic Oscillator}

Let $K=\left(\begin{array}{cc}0 & -1 \\ -1 & 0\end{array}\right)$. For instance, we can choose $\alpha=\beta_{1}=\beta_{2}=0, \phi=\pi$. Then the boundary conditions (32) reduce to

$$
f(-0)=f(+0) \quad \text { and } \quad f^{\prime}(-0)=f^{\prime}(+0) .
$$

Hence $C_{K}=A$ is the classical harmonic oscillator.

\subsection{Boundary Conditions such that $C_{K}=B_{\theta}$}

Let $\beta_{1}=\beta_{2}=0, \alpha \in(0, \pi), \phi=\alpha+\pi / 2$. Then

$$
K=\mathrm{ie}^{\mathrm{i} \alpha}\left(\begin{array}{cc}
\sin \alpha & \cos \alpha \\
\cos \alpha & -\sin \alpha
\end{array}\right)
$$

and the boundary conditions (36) become

$$
\begin{aligned}
0= & \left(1-\mathrm{ie} \mathrm{i}^{\mathrm{i} \alpha} \sin \alpha\right) f(-0)+\mathrm{i}\left(1+\mathrm{ie}^{\mathrm{i} \alpha} \sin \alpha\right) f^{\prime}(-0) \\
& -\mathrm{e}^{\mathrm{i} \alpha} \cos \alpha\left(f(+0)+\mathrm{i} f^{\prime}(+0)\right), \\
0= & -\mathrm{ie}^{\mathrm{i} \alpha} \cos \alpha\left(f(-0)-\mathrm{i} f^{\prime}(-0)\right) \\
& +\mathrm{i}\left(1-\mathrm{ie}^{\mathrm{i} \alpha} \sin \alpha\right) f(+0)+\left(1+\mathrm{ie} \mathrm{e}^{\mathrm{i} \alpha} \sin \alpha\right) f^{\prime}(+0),
\end{aligned}
$$

which, for $\alpha \neq \pi / 2$ is true if and only if

$$
f(-0)=f(+0)=: f(0) \quad \text { and } \quad f^{\prime}(-0)-f^{\prime}(+0)=2 \tan \alpha f(0)
$$

Choose $\theta \in[0, \pi)$ such that $\cot \theta=-\sqrt{2} \tan \alpha$. Then $C_{K}=B_{\theta}$ with $K$ as above. For $\alpha=\pi / 2$, the conditions (37) are equivalent to $f(+0)=f(-0)=0$. 


\subsection{Boundary Conditions with Continuous Derivative}

Let $\alpha \in(0, \pi)$ and let $\beta_{1}=\beta_{2}=0, \phi=\pi / 2-\alpha$. Note that $\mathrm{e}^{\mathrm{i} \phi}=\mathrm{ie}{ }^{-\mathrm{i} \alpha}$ and

$$
K=\mathrm{ie}^{-\mathrm{i} \alpha}\left(\begin{array}{cc}
\sin \alpha & \cos \alpha \\
\cos \alpha & -\sin \alpha
\end{array}\right) .
$$

Then equations (36) become

$$
\begin{aligned}
0= & \mathrm{e}^{-\mathrm{i} \alpha} \cos \alpha f(-0)+\mathrm{i}\left(1+\mathrm{ie}^{-\mathrm{i} \alpha} \sin \alpha\right) f^{\prime}(-0) \\
& -\mathrm{e}^{-\mathrm{i} \alpha} \cos \alpha\left(f(+0)+\mathrm{i} f^{\prime}(+0)\right) \\
0= & -\mathrm{e}^{-\mathrm{i} \alpha} \cos \alpha\left(\mathrm{i} f(-0)+f^{\prime}(-0)\right) \\
& +\mathrm{ie}^{-\mathrm{i} \alpha} \cos \alpha f(+0)+\left(1+\mathrm{ie}^{-\mathrm{i} \alpha} \sin \alpha\right) f^{\prime}(+0) .
\end{aligned}
$$

If $\alpha \neq \pi / 2$, then (39) is equivalent to

$$
f^{\prime}(-0)=f^{\prime}(+0)=: f^{\prime}(0) \text { and } \quad f(+0)-f(-0)=-2 \tan \alpha f^{\prime}(0) .
$$

If $\alpha=\pi / 2$, then (39) is equivalent to $f^{\prime}(-0)=f^{\prime}(+0)=0$.

\section{Interpretation of Some Extensions Via $\delta$ - and $\delta^{\prime}$-Type Interactions}

Observe that the operator $A$ from (3) is closed. Hence the set $H_{+}:=\mathcal{D}(A)$ becomes a Hilbert space with the norm

$$
\|f\|_{+}:=\|f\|_{A}:=\left(\|f\|^{2}+\|A f\|^{2}\right)^{\frac{1}{2}}, \quad f \in H_{+} .
$$

Let $H_{0}:=L_{2}(\mathbb{R})$. In addition to the usual norm on $H$, define

$$
\|f\|_{-}:=\sup \left\{|\langle f, g\rangle|: g \in H_{+},\|g\|_{+} \leq 1\right\}, \quad f \in H_{0},
$$

and we define $H_{-}$to be the closure of $H_{0}$ with respect to the norm $\|\cdot\|_{-}$. Then $\left(H_{-},\|\cdot\|_{-}\right)$ is a Hilbert space and it can be viewed as the dual space of $H_{+}$. Observe that we have the continuous inclusions

$$
H_{+} \subset H_{0} \subset H_{-} .
$$

On says that $H_{0}$ is rigged by $H_{+}$and $H_{-}$, see, for instance, [2], Chapter 14.

If $T: H_{+} \rightarrow H_{0}$ is a bounded linear operator, then define its adjoint operator $T^{*}$ : $H_{0} \rightarrow H_{-}$as the unique bounded linear operator that satisfies

$$
\langle T f, g\rangle=\left\langle f, T^{*} g\right\rangle, \quad f \in H_{+}, g \in H_{0},
$$

where $\langle\cdot, \cdot\rangle$ denotes the inner product on $H_{0}$.

Let us define the functions

$$
w_{1}(t)=\left\{\begin{array}{ll}
v(t, 0), & \text { if } t>0, \\
v(-t, 0), & \text { if } t<0,
\end{array} \quad w_{2}(t)= \begin{cases}v(t, 0), & \text { if } t>0 \\
-v(-t, 0), & \text { if } t<0\end{cases}\right.
$$


with $v$ as in (25). Clearly $w_{1}, w_{2} \in H_{0} \subset H_{-}$. Observe that

$$
\begin{aligned}
w_{1}(+0) & =w_{1}(-0)=w_{2}(+0)=-w_{2}(-0)=v(0,0)=G(0) \\
\text { and } \quad w_{1}^{\prime}(+0) & =-w_{1}^{\prime}(-0)=w_{2}^{\prime}(+0)=w_{2}^{\prime}(-0)=v^{\prime}(0,0)=-1 .
\end{aligned}
$$

Lemma 7. The linear functionals

$$
\begin{gathered}
\delta: H_{+} \rightarrow \mathbb{C}, \quad \delta f=f(0), \\
\delta^{\prime}: H_{+} \rightarrow \mathbb{C}, \quad \delta^{\prime} f=f^{\prime}(0)
\end{gathered}
$$

are bounded and

$$
\delta f=\frac{1}{2}\left\langle A f, w_{1}\right\rangle, \quad \delta^{\prime} f=\frac{1}{2 G(0)}\left\langle A f, w_{2}\right\rangle, \quad f \in H_{+} .
$$

Proof. Note that for any $f \in H_{+}=\mathcal{D}(A)$ and $j=1,2$, using integration by parts twice, we have

$$
\begin{aligned}
\left\langle A f, w_{j}\right\rangle= & \int_{-\infty}^{+\infty}(A f)(t) \cdot w_{j}(t) \mathrm{d} t \\
= & \int_{-\infty}^{0}\left(-f^{\prime \prime}(t)+t^{2} f(t)\right) w_{j}(t) \mathrm{d} t+\int_{0}^{+\infty}\left(-f^{\prime \prime}(t)+t^{2} f(t)\right) w_{j}(t) \mathrm{d} t \\
= & \int_{-\infty}^{0} f(t)\left(-w_{j}^{\prime \prime}(t)+t^{2} w_{j}(t)\right) \mathrm{d} t+\int_{0}^{+\infty} f(t)\left(-w_{j}^{\prime \prime}(t)+t^{2} w_{j}(t)\right) \mathrm{d} t \\
& +f^{\prime}(0)\left\{w_{j}(+0)-w_{j}(-0)\right\}+f(0)\left\{w_{j}^{\prime}(-0)-w_{j}^{\prime}(+0)\right\} \\
= & f^{\prime}(0)\left\{w_{j}(+0)-w_{j}(-0)\right\}+f(0)\left\{w_{j}^{\prime}(-0)-w_{j}^{\prime}(+0)\right\},
\end{aligned}
$$

so the second claim follows from (41). Now the boundedness of $\delta$ and $\delta^{\prime}$ is clear, since $|\delta f|=\left|\frac{1}{2}\left\langle A f, w_{1}\right\rangle\right| \leq \frac{1}{2}\|A f\|\left\|w_{1}\right\| \leq \frac{1}{2}\|f\|_{+}\left\|w_{1}\right\|$, and analogously $\left|\delta^{\prime} f\right| \leq \frac{1}{2 G(0)}\|f\|_{+}\left\|w_{1}\right\|$.

Recall that in our case, $H_{+} \subset \mathcal{D}\left(A_{0}^{*}\right) \subset H_{0} \subset H_{-}$. By definition of $H_{+}$, the operator

$$
\widetilde{A}: H_{+} \rightarrow H_{0}, \quad \widetilde{A} f=A f
$$

is bounded. Let us calculate how $\widetilde{A}^{*}$ acts on elements $g \in \mathcal{D}\left(A_{0}^{*}\right)$. As in the proof of Lemma 7, integration by parts gives for $f \in H_{+}$

$$
\begin{aligned}
\langle A f, g\rangle & =\{\bar{g}(+0)-\bar{g}(-0)\} f^{\prime}(0)+\left\{\bar{g}^{\prime}(-0)-\bar{g}^{\prime}(+0)\right\} f(0)+\left\langle f, A_{0}^{*} g\right\rangle \\
& =\{\bar{g}(+0)-\bar{g}(-0)\} \frac{1}{2 G(0)}\left\langle A f, w_{2}\right\rangle+\left\{\bar{g}^{\prime}(-0)-\bar{g}^{\prime}(-0)\right\} \frac{1}{2}\left\langle A f, w_{1}\right\rangle+\left\langle f, A_{0}^{*} g\right\rangle \\
& =\{\bar{g}(+0)-\bar{g}(-0)\} \frac{1}{2 G(0)}\left\langle f, \widetilde{A}^{*} w_{2}\right\rangle+\left\{\bar{g}^{\prime}(-0)-\bar{g}^{\prime}(+0)\right\} \frac{1}{2}\left\langle f, \widetilde{A}^{*} w_{1}\right\rangle+\left\langle f, A_{0}^{*} g\right\rangle .
\end{aligned}
$$


So by Lemma 7 , we obtain

$$
\widetilde{A}^{*} g=\frac{\bar{g}(+0)-\bar{g}(-0)}{2 G(0)} \widetilde{A}^{*} w_{2}+\frac{\overline{g^{\prime}}(-0)-\overline{g^{\prime}}(+0)}{2} \widetilde{A}^{*} w_{1}+A_{0}^{*} g
$$

or, if we identify $H_{-}$and $\left(H_{+}\right)^{\prime}$,

$$
\widetilde{A}^{*} g=\{\bar{g}(+0)-\bar{g}(-0)\} \delta^{\prime}-\left\{\overline{g^{\prime}}(+0)-\overline{g^{\prime}}(-0)\right\} \delta+A_{0}^{*} g \in\left(H_{+}\right)^{\prime} .
$$

Hence $A_{0}^{*}$ can be seen as a perturbation of $\widetilde{A}^{*}$ :

$$
A_{0}^{*} g=\widetilde{A}^{*} g-\{\bar{g}(+0)-\bar{g}(-0)\} \delta^{\prime}+\left\{\overline{g^{\prime}}(+0)-\overline{g^{\prime}}(-0)\right\} \delta \in\left(H_{+}\right)^{\prime}
$$

for $g \in \mathcal{D}\left(A_{0}^{*}\right)$. Recall that the operators $B_{\theta}$ from Section 2 and $C_{K}$ from Section 3 satisfy $B \subset B_{\theta} \subset A_{0}^{*}$ and $C \subset C_{K} \subset A_{0}^{*}$. So we obtain the following:

- Any function $g \in \mathcal{D}\left(C_{K}\right)$ with $K$ as in Subsection 3.1 satisfies $g(-0)=g(+0)$ and $g^{\prime}(-0)=g^{\prime}(+0)$, hence

$$
C_{K} g=\widetilde{A}^{*} g=A_{0}^{*} g
$$

- Any function $g \in \mathcal{D}\left(C_{K}\right)$ with $K$ as in Subsection 3.2 and $\alpha \neq \pi / 2$ satisfies $g(-0)=$ $g(+0)$ and $g^{\prime}(-0)-g^{\prime}(+0)=2 \tan \alpha g(0)$, hence

$$
C_{K} g=A_{0}^{*} g=\widetilde{A}^{*} g-2 \tan \alpha g(0) \delta .
$$

If we take $\theta$ such that $\cot \theta=-\sqrt{2} \tan \alpha$, we obtain

$$
B_{\theta} g=C_{K} g=\sqrt{2} \cot \theta g(0) \delta+\widetilde{A}^{*} g .
$$

Note that $B_{\theta}$ has exactly one negative eigenvalue if $\theta \in\left(\pi / 2+\alpha_{A}, \pi\right)$ and this eigenvalue decreases monotonically to $-\infty$ as $\theta \rightarrow \pi$, that is $\sqrt{2} \cot (\theta) \rightarrow-\infty$.

- Any function $g \in \mathcal{D}\left(C_{K}\right)$ with $K$ as in Subsection 3.3 satisfies $g^{\prime}(-0)=g^{\prime}(+0)$ and $g(+0)-g(-0)=-2 \tan \alpha g^{\prime}(0)$. Hence, for $\alpha \neq \pi / 2$

$$
C_{K} g=A_{0}^{*} g=\widetilde{A}^{*} g+2 \tan \alpha g^{\prime}(0) \delta^{\prime}
$$

\section{Closing Remarks}

The 3-dimensional point potential for the Schrödinger operator was considered by Zeldovich [16] and Berezin and Faddeev [3]. The free Schrödinger operator with 1dimensional singular potential at 0 was investigated by Šeba in [11] and then later by Kurasov in [10]. Both use von Neumann's extension theory to obtain selfadjoint extensions of a given differential operator on $\mathbb{R} \backslash\{0\}$ and interpret their results in terms of $\delta$ - and $\delta^{\prime}$-interactions at 0 . See [1] for more information on this direction. The 1dimensional harmonic oscillator with $\delta$-interaction at the origin was considered for instance by Gadella, Glasser and Nieto in [7] and Viana-Gomes and Peres in [13]. In both works the eigenfuntions are calculated in terms of confluent hypergeometric functions. Moreover, it is shown that the eigenvalues with odd eigenfunctions are not changed, whereas the 
eigenvalues with even eigenfunctions increase (for $c>0$ ) or decrease (for $c<0$ ) when compared with the eigenvalues of the harmonic oscillator without singular perturbation.

Acknowledgment. We are grateful to the "Fondo de Investigaciones de la Facultad de Ciencias de la Universidad de los Andes, Convocatoria 2014-1 para la Financiación de proyectos de Investigación Categoría: Profesores De Planta ", project "Operadores lineales en espacios con producto interno indefinido", for its financial support.

\section{References / Литература}

1. Albeverio S., Gesztesy F., Høegh-Krohn R., Holden H. Solvable Models in Quantum Mechanics. AMS Chelsea Publishing, Providence, RI, 2005.

2. Berezansky Y.M., Sheftel Z.G., Us G.F. Functional Analysis. Vol. II, Volume 86 of Operator Theory: Advances and Applications. Birkhäuser Verlag, Basel, 1996.

3. Berezin F.A., Faddeev L.D. A Remark on Schrödinger's Equation with a Singular Potential. Soviet Mathematics. Doklady, 1961, no. 2, pp. 372-375.

4. Chernoff P.R., Hughes R.J. A New Class of Point Interactions in One Dimension. Journal of Functional Analysis, 1993, vol. 111, no. 1, pp. 97-117. DOI: 10.1006/jfan.1993.1006

5. Eastham M.S.P. The Asymptotic Solution of Linear Differential Systems. Applications of the Levinson Theorem. Oxford, Clarendon Press, 1989.

6. Gadella M., Glasser M.L., Nieto L.M. The Infinite Square Well with a Singular Perturbation. International Journal of Theoretical Physics, 2011, vol. 50, no. 7, pp. 2191-2200. DOI: $10.1007 / \mathrm{s} 10773-011-0690-5$

7. Gadella M., Glasser M.L., Nieto L.M. One Dimensional Models with a Singular Potential of the Type $-\alpha \delta(x)+\beta \delta^{\prime}(x)$. International Journal of Theoretical Physics, 2011, vol. 50, no. 7, pp. $2144-2152$.

8. Gohberg I., Lancaster P., Rodman L. Indefinite Linear Algebra and Applications. Birkhäuser Verlag, Basel, 2005.

9. Kato T. Perturbation Theory for Linear Operators. Die Grundlehren der mathematischen Wissenschaften, Band 132. New York, Springer-Verlag New York, 1966.

10. Kurasov P. Distribution Theory for Discontinuous Test Functions and Differential Operators with Generalized Coefficients. Journal of Mathematical Analysis and Applications, 1996, vol. 201, no. 1, pp. 297-323. DOI: 10.1006/jmaa.1996.0256

11. Šeba P. The Generalized Point Interaction in One Dimension. Czechoslovak Journal of Physics $B$, 1986, vol. 36, no. 6, pp. 667-673. DOI: $10.1007 /$ BF 01597402

12. Triebel H. Higher Analysis. Hochschulbücher für Mathematik. [University Books for Mathematics]. Johann Ambrosius Barth Verlag GmbH, Leipzig, 1992.

13. Viana-Gomes J., Peres N.M.R. Solution of the Quantum Harmonic Oscillator Plus a DeltaFunction Potential at the Origin: the Oddness of its Even-Parity Solutions. European Journal of Physics, 2011, vol. 32, no. 5, pp. 1377-1384. DOI: 10.1088/0143-0807/32/5/025

14. Weidmann J. Linear Operators in Hilbert Spaces, Volume 68 of Graduate Texts in Mathematics. New York, Berlin, Springer-Verlag, 1980.

15. Weidmann J. Spectral Theory of Ordinary Differential Operators, Volume 1258 of Lecture Notes in Mathematics. Berlin, Springer-Verlag, 1987. 
16. Zeldovic Ya.B. Scattering by a Singular Potential in Perturbation Theory and in the Momentum Representation. Soviet Physics. Journal of Experimental and Theoretical Physics, 1960, no. 11, pp. 594-597.

Received June 17, 2015

УДК $517.921 .25+517.984 .5$

DOI: $10.14529 / \mathrm{mmp} 160106$

\section{ОДНОМЕРНЫЙ ГАРМОНИЧЕСКИЙ ОСЦИЛЯТОР С СИНГУЛЯРНЫМ ВОЗМУЩЕНИЕМ}

\section{В.A. Штраус, М.A. Винклъмайер}

В настоящей работе исследуется одномерный возмущенный гармонический осциллятор с лево-правосторонними граничными условиями в нуле. На рассматриваемый объект можно смотреть как на классический самосопряженный гармонический осциллятор с сингулярным возмущением, сосредоточенным в одной точке. Указанное возмущение порождается дельта-функцией Дирака и/или ее производной. Описываются все самосопряженные реализации этой схемы в терминах указанных граничных условий. Показывается, что при некоторых ограничениях на возмущение (или, что эквивалентно, на граничные условия) у соответствующего дифференциального оператора может появиться ровно одно неположительное собственное значение, и приводится аналитическое выражение для соответствующей собственной функции. Указанное собственное значение пробегает всю неотрицательную полуось когда коэффициент возмущения пробегает установленный промежуток. Для некоторых случаев приводится непосредственная зависимость между подходящими граничными условиями, соответствующим неотрицательным собственным значением и его собственной функцией.

Ключевые слова: гармонический осциллятор; сингулярные возмущения; самосопряженные расширения; отрицательные собственные значения.

Владимир Абрамович Штраус, доктор физико-математических наук, профессор, департамент чистой и прикладной математики, университет Симон Боливар, Каракас, Венесуэла, str@usb.ve.

Моника Винкльмайер, доктор естественных наук, доцент, департамент математики, университет Лос Андес, Богота, Колумбия, mwinklme@uniandes.edu.co.

Поступила в редакцию 17 июня 2015 г.

Вестник ЮУрГУ. Серия «Математическое моделирование

и програм мирование» (Вестник ЮУрГУ ММП). 2016. Т. 9, № 1. С. 73-91 